\title{
La influencia de la ideología en la política turística española y sus enfoques
}

\author{
Alejandro Sepúlveda Sánchez* \\ Universidad Antonio de Nebrija (España) \\ David Airey** \\ University of Surrey (UK)
}

\begin{abstract}
Resumen: Este artículo pretende determinar el enfoque de las políticas turísticas españolas en la etapa democrática y establecer la orientación ideológica de dichas políticas. En cuanto a la metodología, se ha empleado un análisis de contenido para determinar el enfoque de las políticas turísticas; y un Método Delphi para determinar su orientación ideológica. Los resultados del análisis de contenido muestran que los distintos planes de turismo cuentan con enfoques diferenciales. Por su parte, los resultados del Método Delphi muestran los diferentes enfoques ideológicos de los planes de turismo, y, en segundo lugar, los expertos identifican relaciones entre conceptos de política turística y su orientación ideológica. Las principales conclusiones son que existen diferencias en el enfoque de las políticas turísticas, pero igualmente, que existen una serie de contenidos comunes en las políticas de turismo de España. Finalmente, esta investigación no puede concluir que exista una relación entre ideología y política turística.
\end{abstract}

Palabras clave: política, turismo, ideología, Partido Socialista Obrero Español (PSOE), Partido Popular (PP), neoliberalismo, social democracia.

\section{The influence of ideology in Spanish tourism policy and its orientation}

Abstract: This article seeks to determine the approach of the Spanish tourism policy in the democratic period and to establish the ideological orientation of the policies. In reference to the methodology, a content analysis has been utilised to determine the approach of the tourism policies; and a Delphi Method has been used to determine the ideological orientation. The results from the content analysis show how the different tourism plans have differential approaches. In addition, the results from the Delphi Method show the different ideological orientations of the tourism plans, and, secondly, the experts identified links between tourism policy concepts and its ideological orientation. The main conclusions are that, there exist differences in the approach of the tourism policies, but at the same time, there exist a series of common contents in the Spanish tourism policies. Finally, this research cannot conclude that a link between ideology and tourism policy may exist.

Keywords: policy, tourism, ideology, Spanish Socialist Workers Party (PSOE), Popular Party (PP), neoliberalism, social democracy.

\section{Introducción}

El sistema turístico español atraviesa en la actualidad momentos de éxito. Probablemente por la coyuntura política y social de los destinos turísticos competidores del mediterráneo oriental, caracterizados en la actualidad por la alta inestabilidad política debido a fenómenos como el terrorismo yihaidista, nuestro país está asistiendo a un periodo de crecimiento turístico interanual espectacular, habiéndose alcanzado, según señalan algunas previsiones, los 65 millones de turistas en 2015. Y en el presente

\footnotetext{
* Consultor de Turismo y Estudiante de Doctorado; E-mail: proyectos@alejandrosepulveda.info

** Emeritus Professor; University of Surrey (UK); E-mail: d.airey1@btinternet.com
} 
ejercicio, según indica la Encuesta de Movimientos Turísticos en Frontera, elaborada actualmente por el INE (Instituto Nacional de Estadística, 2016), durante los cuatro primeros meses del presente ejercicio habrían llegado a España 18.104.490 turistas, lo que significa un crecimiento del 13\% con respecto al mismo periodo de 2015, por lo que se están superando las previsiones de todos los organismos oficiales.

Una vez contextualizada la actualidad del turismo español, se debe señalar que un crecimiento turístico de la envergadura que se ha mencionado anteriormente, y en una dinámica empresarial y tecnológica que evoluciona a un ritmo vertiginoso, necesita de políticas y programas públicos para regular el turismo. Uno de los motivos es que el turismo, además de ser una actividad económica, tiene un elevado impacto medioambiental y territorial. Pero todavía más importante, es que como bien señala el Manual de Modelos de Gestión Turística Local de la Federación Española de Municipios y Provincias (FEMP), y la Secretaría General de Turismo (2008), existe el concepto de producto turístico global, con el cual sus autores se refieren a que la experiencia turística requiere de servicios no sólo privados, como alojamiento, restauración y transporte; sino también de servicios de provisión pública como son el abastecimiento energético, de recursos hídricos, los servicios sanitarios y la seguridad ciudadana, entre otros, y que dependen directamente de las administraciones locales, responsables de la gestión del turismo en destino.

Por todos los motivos expuestos con anterioridad, se puede afirmar la necesidad de desarrollar procesos de planificación turística en todos los niveles administrativos del Estado con competencias en turismo, y, por consiguiente, la aplicación de una determinada política turística que en un marco de cooperación público-privada defina los ejes estratégicos y los programas de actuación correspondientes para un marco temporal determinado y un territorio concreto. De esta forma, una vez razonada la necesidad de una política turística pública, se cuestiona ahora cuáles son las bases ideológicas de los diferentes partidos políticos a la hora de enfocar y dirigir los procesos de planificación pública del turismo, de cara a determinar si la ideología de los partidos influye en el desarrollo de las políticas turísticas, y en última instancia, si se pueden identificar diferencias significativas entre las políticas aplicadas desde el Estado por los dos principales partidos políticos españoles en la etapa democrática. A continuación, se detallan todos los planes de turismo que se han aprobado desde el Gobierno de España tomando como marco temporal el periodo 1992-2015 en el Cuadro 1.

- Plan Marco de Competitividad del Turismo Español - FUTURES I 1992-1995.

- Plan Marco de Competitividad del Turismo Español - FUTURES II 1996-1999.

- Plan Integral de Calidad del Turismo Español - PICTE 2000-2006.

- Plan del Turismo Español Horizonte 2020.

- Plan Nacional e Integral de Turismo (PNIT) 2012-2015.

\section{Cuadro 1. Línea temporal con la evolución histórica de la política turística española en la etapa democrática}

199219931994199519961997199819992000200120022003200420052006200720082009201020112012201320142015

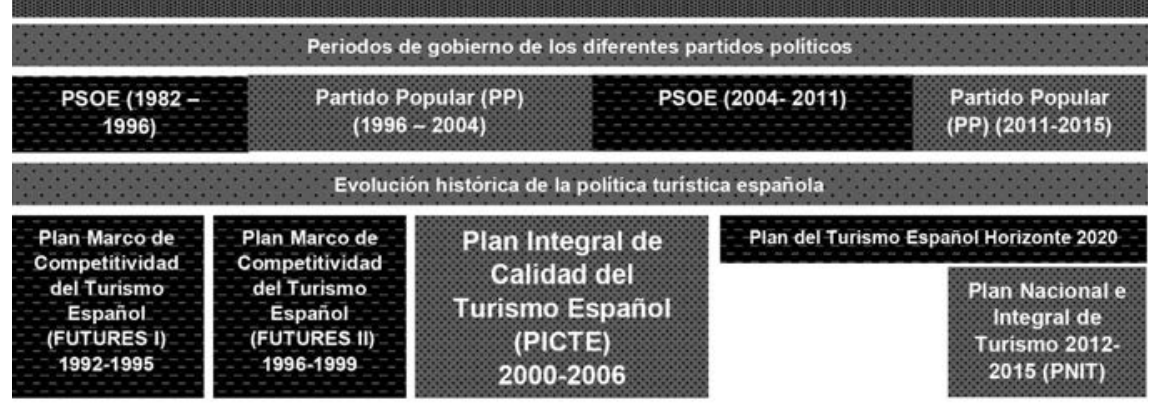

Planes de turismo aprobados por el Partido Socialista Obrero Español (PSOE)

Planes de turismo aprobados por el Partido Popular (PP)

Fuente: elaboración propia 
Como conclusión a esta breve introducción, se puede mencionar que la evolución de la política turística española ha sido investigada por numerosos autores (Correyero, 2004; Pellejero, 2004a; Pellejero, 2004b; Velasco, 2005), y dichos estudios han incluido una aproximación y consideración de la ideología. Sin embargo, ninguna de las investigaciones anteriores ha focalizado su objeto de estudio en la influencia de la ideología en el desarrollo de las estrategias nacionales de turismo en España. Consecuentemente, se destaca ahora la necesidad de una investigación integral sobre la influencia de la ideología en la política turística española, lo que permitirá una mejor comprensión del desarrollo de las políticas en el ámbito del turismo, tanto para las administraciones públicas, la industria turística, los partidos políticos y la sociedad española.

\section{Objeto de estudio}

Una vez presentada la introducción y explicados los motivos principales que justifican esta investigación, se dedica ahora un apartado a determinar el objeto de estudio. De esta forma, el objeto de investigación de este proyecto ha quedado definido de la siguiente forma:

- Determinar el contenido y enfoque de los principales documentos de política turística estatal en la etapa democrática y la distribución de las diferentes ideologías en cada uno de los documentos estratégicos de turismo de los diferentes partidos políticos.

\section{Marco teórico}

\subsection{Ideología y política turística}

Ladd (1989, p. 11) define la ideología como "un conjunto de creencias políticas y valores que están relacionados entre sí...”. Haciendo especial referencia al turismo, Hall y Jenkins (1995, p. 69) definen la ideología como "un conjunto de valores que definen los parámetros dentro de los cuales se definen y discuten los problemas y se conciben y llevan a cabo las soluciones". Todos estos argumentos reflejan la importancia de la ideología en la configuración del contexto para las decisiones políticas sobre turismo, un aspecto mencionado por Elliot (1997, p. 17) que sugiere que "la gestión del turismo dependerá de la cultura política del país y de la ideología de su gobierno". Asimismo, Zhang y Yang destacan que el turismo (2009, p. 163) "es ampliamente reconocido como un aspecto positivo y es bienvenido por todas las ideologías políticas alrededor del mundo".

Dredge y Jenkins (2007) hicieron una revisión histórica de políticas públicas e ideologías, citando a autores tan importantes en el pensamiento económico y político como Adam Smith y Carlos Marx. Adam Smith argumentaba que "las economías de mercado por su cuenta, sin intervención del gobierno, tienden a alcanzar el equilibrio - lo que se denomina la mano invisible -" (Dredge y Jenkins, 2007, p. 77). Por el contrario, Carlos Marx rechazó en sus teorías la noción de los mercados libres definidos por Adam Smith. Adicionalmente, Marx ha sido muy influyente en el desarrollo de la ideología de los partidos socialistas en los tiempos modernos, que han evolucionado desde los principios del socialismo marxista, hacia una nueva ideología que ha recibido el nombre de social democracia. Asimismo, Dredge y Jenkins (2007) mencionan como Keynes propuso una amplia intervención de los gobiernos en la economía. Adicionalmente, Webster e Ivanov (2016) identificaron cinco grandes ideologías políticas en el ámbito del turismo diferenciando aquellas que ponen el énfasis en la provisión y regulación del Estado, y aquellas que resaltan la importancia de las fuerzas del mercado.

En contraposición a las ideologías socialistas, Harvey (2007, p. 22) argumenta que el neoliberalismo es una teoría de política económica mediante la cual el bienestar social se puede conseguir de mejor forma mediante la "maximización de la libertad empresarial con un marco institucional caracterizado por los derechos de propiedad privada, mercados sin responsabilidades y el libre comercio". Según los argumentos de Harvey (2007), el neoliberalismo ha fallado en su intento de promover el crecimiento económico mundial, pero ha sido sin embargo un gran éxito en la restauración de la posición social de las clases dirigentes.

A continuación, se realiza una revisión de las principales definiciones del concepto de política. Airey y Chong (2011, p. 42) dicen que "no existe una definición universalmente aceptada". Dichos autores 
sugieren que la definición de política más simple fue la proporcionada por Dye cuando describe que política es lo "que los gobiernos eligen hacer o no hacer" (Dye, 1978; citado por Dredge y Jenkins, 2007, p.5).

La política turística es un fenómeno complejo debido a que requiere la participación de los gobiernos, la industria turística, los clientes, la comunidad local y otras organizaciones turísticas y sociales, y, por lo tanto, debe recibir atención por parte de los investigadores turísticos, de los partidos políticos, las empresas y destinos turísticos y de la sociedad en general. Sin embargo, de acuerdo con Dredge y Jenkins (2007, p. 13) "todavía el análisis del quehacer político en turismo es muy reducido". En este sentido, se puede argumentar que la política turística ha sido un área menos estudiada que otros aspectos del turismo. La razón de la falta de atención hacia la política turística podría ser, de acuerdo con Dredge y Jenkins (2007), que el turismo es un área relativamente nueva de intervención del gobierno.

Por su parte, Fayos-Sola (1996) explica en su investigación cómo el turismo ha evolucionado de un modelo masivo de producción a la Nueva Era del Turismo. Realizando una clasificación de nuevas políticas turísticas, Fayos-Sola (1996) distingue tres generaciones de política turística. La primera generación se caracteriza por el objetivo de incrementar el número de visitantes y estimular el turismo masivo. Fayos-Sola (1996, p. 408) señala cómo las dificultades económicas en 1970 y a comienzos de 1980 dieron lugar a una segunda generación de política turística donde "los impactos sociales, económicos y medioambientales de la actividad turística son mejor entendidos". Sin embargo, la aparición de la Nueva Era del Turismo estableció la tercera generación de política turística que se basa en promover la competitividad de la industria turística.

Una vez analizados los aspectos específicos de la política turística y su relación con las ideologías y los cambios de gobierno, Dredge y Jenkins (2007) señalan los diferentes enfoques de planificación turística identificados por Getz (1986) y Hall (1998), y los critican argumentando que la mejor forma de entender la evolución de la política turística es tomar en consideración la importancia de los cambios ideológicos, generalmente relacionados con el cambio de partido político en el gobierno. De hecho, la principal hipótesis de esta investigación, es que la ideología ha influido en el desarrollo de las políticas turísticas nacionales en España y que los diferentes partidos políticos han asumido diferentes enfoques de la política turística, aunque se deberá determinar mediante la metodología el grado de influencia de las ideologías, así como las diferencias en los enfoques y contenidos. Dicha hipótesis se apoya en los argumentos de los investigadores Dredge y Jenkins (2007, p. 226) cuando afirman que "la política turística pública refleja la ideología del gobierno". Adicionalmente, la hipótesis de esta investigación también está apoyada por las afirmaciones del estudio llevado a cabo por Wang y Ap (2013, p. 223), quienes citando a Elliot (1997) y Pearce (1992) establecieron cómo "la ideología de un gobierno que refleja la cultura política nacional y las preferencias de los partidos políticos determinan el estilo de la administración turística pública”.

\subsection{La política turística española: una revisión histórica}

La Guerra Civil entre el bando republicano y el bando nacional paralizó las llegadas turísticas a nuestro país, así como las actividades del turismo doméstico de los ciudadanos españoles (Pellejero, 2004a). Cuando el conflicto civil terminó en 1939, la política turística aplicada por el Gobierno de Franco fue claramente intervencionista (Pellejero, 2004a). Asimismo, el gobierno entendió que el turismo podía ser una de las principales industrias que contribuyeran a la recuperación económica del país después de la Guerra Civil, gracias a la entrada de divisas (Correyero, 2004; Pellejero, 2004a).

España, bajo el Gobierno de Franco, estaba completamente aislada económica y políticamente en el escenario internacional. Sin embargo, entre 1951 y 1962 es importante mencionar que el aislamiento internacional del Gobierno de Franco se redujo significativamente (Pellejero, 2004a). En relación con la política turística, el gobierno creó el Ministerio de Información y Turismo en 1951, y un organismo autónomo denominado Administración Turística Española (Pellejero, 2004b). Incluso más importante se considera señalar la aprobación del Plan Nacional de Turismo en 1953 (Pellejero, 2004b). Dada la importancia del turismo en España, el régimen aplicó un enfoque intervencionista (Pellejero, 2004a; Pellejero, 2004b; Velasco, 2005) y una ideología centralista a la política turística (Ivars, 2004; Pellejero, 2004a; Pellejero, 2004b; Velasco, 2005).

No obstante, el Gobierno de Franco no reconoció las potenciales amenazas del turismo, y esto se relaciona con uno de los cuatro enfoques de la planificación turística definidos por Getz (1987) citado por 
Ivars (2004, p. 317), quien define esta situación mediante el término anglosajón boosterism que significa una "evaluación exenta de críticas hacia el turismo identificándolo como un fenómeno intrínsecamente positivo e ignorando sus potenciales efectos negativos". Dicho enfoque de la planificación turística asumido por el Gobierno de Franco ha creado algunos de los problemas medioambientales, sociales y de saturación que algunos destinos turísticos españoles siguen sufriendo en la actualidad.

En 1962 el Banco Mundial publicó un informe sobre la economía española incluyendo una sección dedicada al turismo (Pellejero, 2004b; Velasco, 2005). El informe argumentaba que la situación geográfica de España, cercana a los principales destinos emisores del norte de Europa, estimularía la expansión del turismo, pero recomendó al Gobierno de Franco algunas acciones para regular su desarrollo (Velasco, 2005). Tras el Informe del Banco Mundial, Ivars (2004), Velasco (2005) y Pellejero (2004b), describen la aprobación de tres Planes de Desarrollo Económico y Social, y cada uno de ellos incluía una sección específica dedicada al turismo, lo cual se relaciona, de acuerdo con los argumentos de Velasco (2005), con la fase de planificación indicativa mencionada por Ivars (2004) cuyo objetivo fue la maximización del crecimiento turístico (Aguiló y Vich i Martorell, 1996; Pearce, 1997; Pellejero, 2004a, Pellejero, 2004b; Velasco, 2005). Asimismo, durante este periodo, el Gobierno de Franco adoptó un enfoque economicista hacia la política turística (Ivars, 2004, p. 317) que "concibe el turismo como un instrumento que puede ayudar a alcanzar ciertos objetivos económicos".

Fayos-Sola (1997, p. 408), como ya se ha analizado, identificó tres generaciones de política turística, y el argumento de la presente investigación es que la política seguida por el gobierno de Franco puede clasificarse dentro de la primera generación, dado que su objetivo es "estimular el turismo de masas desde el punto de vista cuantitativo". Asimismo, como bien explica Ivars (2004), existió un elemento de especulación territorial y una ausencia de planificación turística específica lo que generó un desarrollo urbanístico masivo.

La muerte de Francisco Franco en 1975 estableció el comienzo de la transición política en España con los gobiernos de UCD bajo la dirección del Expresidente del Gobierno Adolfo Suárez. Durante los últimos años del régimen, la política turística expansiva estaba alcanzado su fin (Velasco, 2005). Los problemas estructurales de los destinos españoles, añadidos a la inestabilidad económica y política, condujeron a la primera crisis del turismo español (Pellejero, 2004b; Velasco, 2005). Dada esta situación, el objetivo básico de la política turística seguida durante esta etapa fue la limitación del desarrollo turístico y la aprobación de varios Planes de Modernización Hotelera (Pellejero, 2004b; Velasco, 2005). Dentro de esta fase, se podría aplicar la teoría de Fayos-Sola (1996, p. 408) argumentando que la política seguida por el gobierno de la transición corresponde a la segunda generación de política turística dado que "los impactos sociales, económicos y medioambientales del turismo son mejor comprendidos". Asimismo, la transición política hacia la democracia llevó a la aprobación de la Constitución Española de 1978, que estableció un sistema político descentralizado, incluyendo las competencias en turismo, con la creación de las Comunidades Autónomas. Asimismo, se produjo una reducción del intervencionismo en el ámbito turístico (Pellejero, 2004a).

En 1982, el candidato del Partido Socialista Obrero Español (PSOE) Felipe González Márquez, consiguió una importante victoria en las elecciones generales con 202 escaños. La transferencia de las competencias en turismo a las Comunidades Autónomas produjo una confusión inicial en el gobierno central, lo que supuso una ausencia de una política turística formal hasta 1992 (Pellejero, 2004a; Velasco, 2005). En términos políticos generales, la política seguida durante los primeros años de los gobiernos socialistas se caracterizó por una reducción de la estructura política del turismo (Ivars, 2004; Velasco, 2005), así como por la privatización de algunas empresas turísticas públicas. Desde 1991, como comentó Pearce (1997), el gobierno socialista comenzó a reconocer las debilidades estructurales del modelo turístico español, lo que llevó a la aprobación en 1992 del Plan Marco de Competitividad del Turismo Español - FUTURES I. Dicho documento de política turística representó el primer paso en la definición de una estrategia turística nacional (Pellejero, 2004a). Asimismo, siguiendo el modelo de generaciones de política turística, se puede relacionar este plan de turismo con la tercera generación de política turística definida por Fayos-Solá (1996, p. 409), en la cual "la competitividad se convierte en el objetivo fundamental".

En 1996 José María Aznar, candidato del Partido Popular (PP), consiguió su primera victoria en las elecciones generales con mayoría simple. El Plan FUTURES II, aprobado por el anterior gobierno 
socialista, estaba dando buenos resultados, y consecuentemente el nuevo gobierno tomó la decisión de mantener la política turística (Velasco, 2005). En el año 2000 el presidente Aznar consiguió su segunda victoria en las elecciones generales, en esta ocasión con mayoría absoluta. En relación con la política turística, el gobierno aprobó el Plan Integral de Calidad del Turismo Español - PICTE 2000-2006, estableciendo la calidad como el principal objetivo de la política (Almeida, 2012; Velasco, 2004; Velasco, 2005).

En relación con la política turística de los nuevos gobiernos socialistas de Zapatero (2004-2011), en 2007, después de un extenso proceso de participación de los stakeholders, el gobierno aprobó el Plan del Turismo Español Horizonte 2020. El nuevo documento de política turística fue firmado y acordado por el Gobierno de España, todas las Comunidades Autónomas, las autoridades locales y el sector privado. El argumento de esta investigación es que el Plan Horizonte 2020 representa una innovación en la revisión histórica de la política turística que se viene realizando, dado que aplica el concepto de participación de todos los agentes del turismo, un alto grado de consenso político y el hecho de que por primera vez los objetivos económicos del plan se presentan alineados con la sostenibilidad del modelo turístico español (Almeida, 2012).

Finalmente, en referencia a la política turística del último gobierno del Partido Popular con Mariano Rajoy como presidente (2011-2016), los conservadores aprobaron un documento de política turística completamente diferente, que recibió el nombre de Plan Nacional e Integral de Turismo 2012-2015 (PNIT). El nuevo documento redujo altamente la participación de los agentes involucrados en el turismo, así como la inclusión de los principios de la sostenibilidad turística. Adicionalmente, la hipótesis de esta investigación es que el documento muestra una clara orientación liberal, con medidas como el estímulo de la demanda turística, el incremento de la unidad de mercado, el fortalecimiento de la visión de España como destino unitario y, asimismo, el documento propuso la introducción de capital privado en Turespaña.

\section{Metodología y desarrollo del estudio}

En relación con el enfoque de investigación, el presente proyecto trabaja con una hipótesis inicial basada en que, efectivamente, y de acuerdo con los contenidos del marco teórico, la ideología ha influido en el desarrollo de las políticas turísticas estatales en España. Asimismo, se considera que existen diferentes contenidos y enfoques de las distintas políticas turísticas, así como múltiples visiones políticas del turismo de los dos principales partidos políticos que han ostentado la responsabilidad del Gobierno de España (PSOE Y PP). Por lo tanto, el presente proyecto de investigación, a pesar de contar con una hipótesis inicial, va a adoptar un enfoque inductivo.

En referencia a los instrumentos y metodologías de investigación, este estudio tiene un carácter combinatorio, pues al tratar de dar respuesta a dos cuestiones diferenciadas, se van a emplear dos metodologías diferentes, como se muestra en el Cuadro 2.

\section{Cuadro 2. Esquema con la metodología de la investigación utilizada}

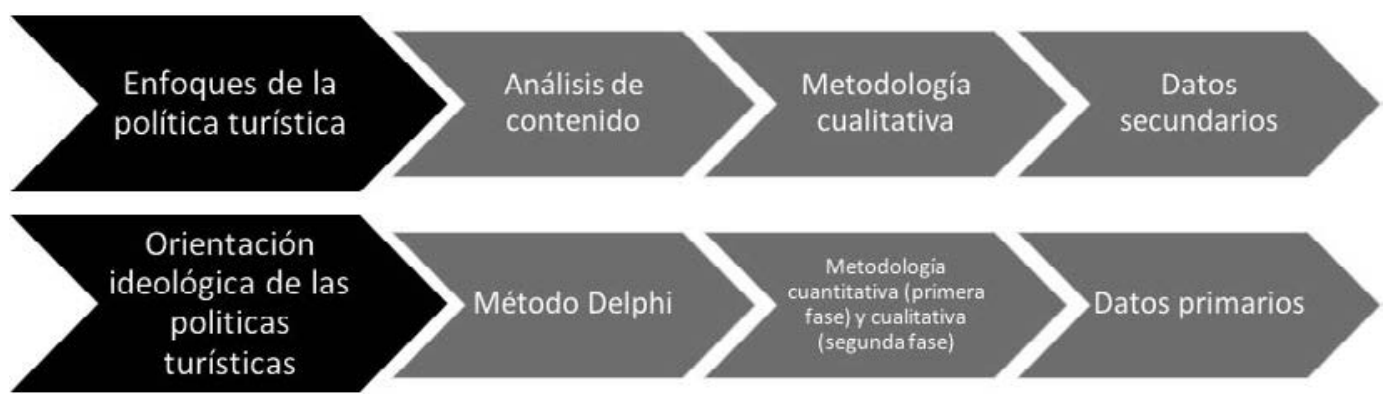

Fuente: elaboración propia 
1- De cara a determinar los enfoques de los diferentes documentos de política turística estatal, la metodología utilizada va a ser el análisis de contenido, una técnica puramente cualitativa, que se basa en un análisis pormenorizado de datos secundarios, que en este caso son los documentos de política turística estatal. Los cuatro documentos de política turística que han sido seleccionados son los siguientes:

- Plan Marco de Competitividad del Turismo Español - FUTURES I 1992-1995 (PSOE).

- Plan Integral de Calidad del Turismo Español - PICTE 2000-2006 (PP).

- Plan del Turismo Español Horizonte 2020 (PSOE).

- Plan Nacional e Integral de Turismo (PNIT) 2012-2015 (PP).

De cara a determinar el enfoque y contenido de los documentos de política turística estatal, el método de análisis de contenido empleado se ha basado en extraer 338 citas textuales de los documentos para después identificar cuál es el concepto o idea principal que se encuentra detrás de cada una de las citas identificadas para cada plan estratégico de turismo. Para una descripción más detallada, los pasos que se han seguido en el análisis de contenido son los siguientes:

1. Obtener los datos secundarios para el análisis. Los datos secundarios fueron facilitados por el Instituto de Turismo de España - Turespaña, que es la Organización Nacional de Turismo en nuestro país $(\mathrm{ONT})$.

2. Leer detenidamente los materiales, que son los cuatro documentos de política turística mencionados. La lectura de los documentos debe ser precisa para identificar citas textuales relevantes que puedan indicar un concepto o enfoque y una posible orientación ideológica de cara al diseño del cuestionario del Método Delphi.

3. Extraer las citas textuales identificadas mediante su procesamiento en un documento de Microsoft Word.

4. Identificar el concepto o enfoque que se esconde detrás de cada cita textual. Este proceso fue de carácter inductivo y se basó en un pensamiento razonado que pudiera llevar a identificar la palabra clave que se encuentra detrás de cada cita textual, para después poder determinar y clasificar el contenido de cada plan de turismo. A continuación, se muestra un ejemplo:

a. Cita textual: "El objetivo del Plan FUTURES es establecer las estrategias necesarias de cara a consolidar la posición del turismo como un sector económico con una fuerte capacidad competitiva".

b. Concepto y enfoque: si leemos detenidamente la cita textual se puede determinar cómo la principal idea está relacionada con la competitividad.

c. Este proceso fue repetido con cada una de las citas textuales de cada plan turístico.

5. Después de finalizar el proceso descrito, se ha diseñado una tabla para cada documento estratégico de turismo que contiene las citas textuales y los conceptos identificados. De cara a la presentación de los resultados del análisis de contenido para determinar el enfoque de las políticas turísticas, los conceptos que surgieron tras la extracción de las citas textuales fueron agrupados en categorías, y posteriormente se construyeron unos gráficos de columnas que muestran los conceptos más repetidos para cada uno de los planes turísticos.

2-De cara a identificar el enfoque ideológico de los diferentes planes estratégicos de turismo y la relación entre ideología y política turística, la metodología empleada va a ser el Método Delphi, una técnica cuantitativa y cualitativa que consta de dos fases fundamentales. En la primera, se ha diseñado un cuestionario consistente en 48 enunciados de política turística extraídos de los datos secundarios obtenidos en la primera fase. En concreto, se seleccionaron 12 enunciados para cada plan turístico, de forma que se mantuviera el equilibrio entre los cuatro planes y el cuestionario no fuese excesivamente extenso. La selección de los enunciados siguió las teorías del muestreo por conveniencia, pues se eligieron aquellos enunciados de política turística que se consideraba tenían una mayor carga ideológica, y, además, de cara a no sesgar los resultados de la primera fase, se pretendió escoger las citas textuales siguiendo un criterio de equilibrio ideológico. Posteriormente, para el diseño del propio cuestionario, se empleó la herramienta Google Forms y las citas textuales fueron ordenadas alfabéticamente, escribiendo al final de cada una de ellas el número correspondiente a cada plan turístico. De esta forma, junto a cada cita textual seleccionada y su número correspondiente, se ofrecían a los expertos cinco opciones de respuesta para clasificar la ideología de cada cita: 

A. Izquierda
B. Social-demócrata
C. Centro liberal
D. Neoliberal
E. Ninguna de las anteriores

En cuanto a la elección de los expertos, se trató de buscar un equilibrio entre el ámbito de la empresa privada del turismo, el ámbito de la docencia y la investigación, el ámbito de la política turística y el ámbito de la administración turística pública del Estado:

- Andrés Fernández Alcantud - Director de Investigación, Desarrollo e Innovación Turística en Segittur. Su excelente perfil corresponde al ámbito de la administración turística estatal.

- Carlos Romero Dexeus - Director en Segittur. Su también excelente perfil corresponde al ámbito de la administración turística estatal.

- David Mora Gómez - Director de Programas Formativos en la Escuela Universitaria del Real Madrid en la Universidad Europea y Consultor de Turismo en Emoturismo. David ha trabajado en la empresa turística privada, fue becario de Turespaña, ha dirigido la División de Turismo de la empresa de consultoría Cegos, es conferenciante y profesor universitario.

- Manuel Figuerola Palomo - Director del Doctorado en Turismo de la Universidad Nebrija. Manuel Figuerola es un referente nacional e internacional en turismo, ha dedicado toda su carrera profesional a fomentar el estudio y la investigación en turismo, y ha participado en el diseño de numerosos desarrollos turísticos.

- Enrique Martínez Cantero - Director y Fundador de Govermamce. Enrique tiene un excelente perfil, no especializado directamente en turismo, pero sí en temáticas relacionadas con el rendimiento de los gobiernos, área de negocio principal de su actual empresa, por lo que su relación y conocimiento del ámbito político es muy amplio y su opinión es altamente reconocida en este proyecto de investigación.

Posteriormente, ya entrando en la segunda fase, se diseñó un segundo cuestionario en formato Word en el cual se presentaban y desvelaban a los expertos los resultados globales de sus respuestas para cada una de las 48 preguntas incluidas. Dado que el objetivo de esta segunda fase de la Metodología Delphi es tratar de alcanzar un mayor nivel de consenso en las respuestas de los expertos participantes, se redactaron preguntas abiertas comentando los resultados de cada uno de los enunciados. Además, con el objetivo de contrastar la hipótesis principal, se incluyó al final del segundo cuestionario una pregunta abierta final sobre la relación entre ideología y política turística.

\section{Resultados de la investigación}

\subsection{Resultados del análisis de contenido}

Con carácter general, la metodología que se ha seguido para convertir los datos secundarios en datos con formato para ser presentados, es realizar un recuento manual del número total de conceptos que han sido identificados para cada plan de turismo, y posteriormente ordenar los conceptos según su frecuencia de repetición.

Comenzando con el análisis del primer documento de política turística considerado en este estudio, el Plan Marco de Competitividad del Turismo Español - Plan FUTURES I (1992-1995), el enfoque principal de este documento es la competitividad, que acumula un total de 15 citas textuales sobre un total de 55 (ver Gráfico 1). El enfoque de competitividad de este primer plan de turismo nacional se relaciona con la pérdida de competitividad que estaba sufriendo el turismo español en los años en los que se aprobó este documento, frente a los destinos competidores del mediterráneo. En segundo lugar, se puede observar cómo tras el análisis de contenido, la categoría de conceptos mercantiles ocupa la segunda posición del enfoque de esta política turística con 11 citas textuales sobre el total. Los conceptos mercantiles son una agrupación de numerosos términos que están relacionados con las disciplinas de economía y administración de empresas, y se ha decidido agruparlos para evitar presentar una elevada dispersión de conceptos. 


\section{Gráfico 1. Enfoque y contenido del Plan Marco de Competitividad del Turismo Español - Plan FUTURES I (1992-1995)}



Fuente: elaboración propia

Siguiendo con el análisis de resultados, el estudio se centra ahora en el Plan Integral de Calidad del Turismo Español-PICTE (2000-2006). La principal orientación de este documento de política turística desde el punto de vista del contenido es la calidad, que se sitúa en primera posición con un total de 44 citas textuales sobre un total de 108 identificadas (ver Gráfico 2). La categoría de conceptos mercantiles, ocupa, a mucha distancia de la primera, la segunda posición del ranking de contenidos elaborado para este plan de turismo con 20 enunciados totales, del mismo modo que en el Plan FUTURES I. Muy de cerca le sigue la categoría de cooperación, que ocupa la tercera posición al igual que en el Plan Marco de Competitividad con un total de 18 citas textuales.

\section{Gráfico 2. Enfoque y contenido del Plan Integral de Calidad del Turismo Español - PICTE 2000-2006}

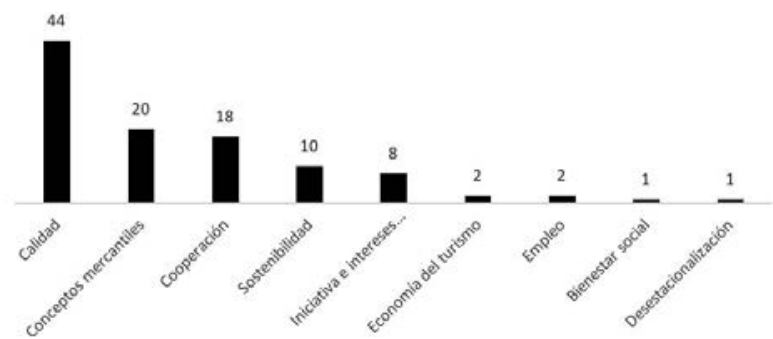

Fuente: elaboración propia

En referencia al Plan del Turismo Español Horizonte 2020, lo primero que debe comentarse es que, a diferencia de los dos planes anteriores, no existe una categoría que ostente la primera posición con gran diferencia, sino que se produce prácticamente un alineamiento entre la importancia de los primeros cuatro conceptos. De esta forma, el primer enfoque en importancia desde el punto de vista cuantitativo, es la sostenibilidad con un total de 16 citas textuales sobre un total de 89 (ver Gráfico 3). No obstante, en segundo lugar, se sitúa el concepto de competitividad con un total de 15 citas textuales, a muy poca distancia por lo tanto del concepto de sostenibilidad. En tercer lugar, aparece el enfoque de bienestar social en las políticas de turismo con un total de 13 citas textuales, pudiendo identificar aquí una diferencia importante con el PICTE del Partido Popular donde el bienestar social se situaba en última posición, y una coherente similitud con las políticas del Partido Socialista. Asimismo, y empatado con el bienestar social, aparece el concepto de participación de stakeholders con un total de 13 citas textuales, lo cual indica el alto grado de compromiso y participación que se alcanzó en la elaboración de esta política turística. Por su parte, los conceptos mercantiles, como agrupación de distintos enfoques de empresa, ocupan la quinta posición en el contenido de este plan con un total de 11 citas textuales, y parece que puede establecerse aqui una cierta continuidad en la presencia de este concepto en las distintas políticas turísticas elaboradas por los diferentes gobiernos. 


\section{Gráfico 3. Enfoque y contenido del Plan del Turismo Español Horizonte 2020}

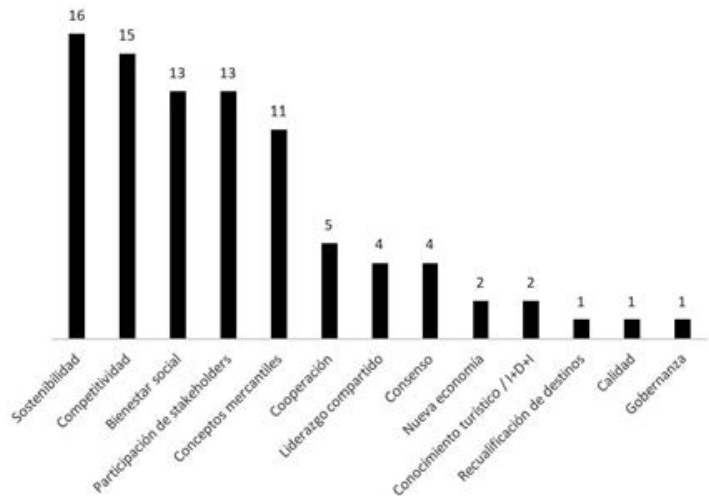

Fuente: elaboración propia

Entrando finalmente al análisis del Plan Nacional e Integral de Turismo 2012-2015 (PNIT), aprobado por el último gobierno del Partido Popular, se puede apreciar un claro cambio de enfoque producido por el cambio de partido en el gobierno, pues a diferencia del Plan Horizonte 2020 donde la sostenibilidad era el principal concepto, en el PNIT los conceptos mercantiles ocupan con mucha diferencia la primera posición del ranking de contenidos, con un total de 28 citas textuales de un total de 87 enunciados (ver Gráfico 4). El siguiente concepto en importancia es la sostenibilidad, con un total de 11 enunciados, y a mucha distancia del primer enfoque. Sin embargo, debe señalarse que desde el comienzo de las políticas turísticas estatales con el Plan FUTURES I en 1992, la sostenibilidad ha sido un concepto que ha ido creciendo en importancia en la política de turismo, alcanzado su máximo exponente en el Plan del Turismo Español Horizonte 2020. No obstante, en el PNIT ocupa la segunda posición, lo cual indica también una relevancia importante en el contenido. La competitividad aparece como el tercer grupo en importancia, con un total de 10 enunciados, y puede deducirse también que se trata de uno de los conceptos más importantes en el histórico de la política turística española. La iniciativa y las necesidades privadas conforman el cuarto puesto con un total de 9 citas textuales, y puede adelantarse en este momento que se trata de un enfoque de política turística propio del Partido Popular.

Gráfico 4. Enfoque y contenido del Plan Nacional e Integral de Turismo 2012-2015 (PNIT)

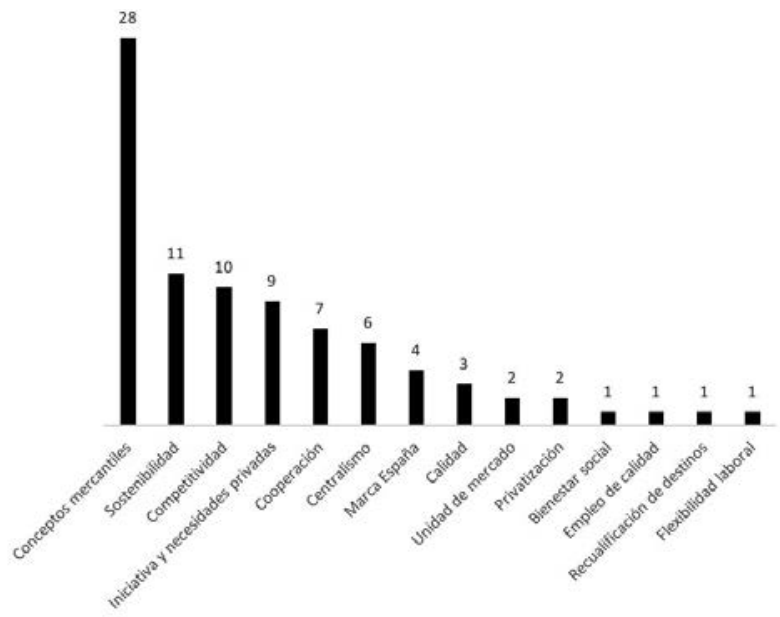

Fuente: elaboración propia 


\subsection{Resultados de la Metodología Delphi}

En referencia a los resultados de la primera fase del Método Delphi, se discute ahora la orientación ideológica del Plan Marco de Competitividad del Turismo Español - FUTURES I (1992-1995). Se debe hacer mención expresa aquí, al hecho de que los resultados de esta sección no son concluyentes, pues están basados en las opiniones de cinco expertos en diferentes ámbitos del turismo, pero no suponen una muestra lo suficientemente amplia como para considerar los resultados como definitivos y extrapolables a una población. Los resultados muestran cómo la ideología social-demócrata, propia del Partido Socialista Obrero Español (PSOE), es la opción más votada con un 45\% de las respuestas (ver Gráfico 5). Asimismo, la opción ideológica de izquierdas ha recibido un $8 \%$ de los votos, lo que sumado a la opción anterior supone un 53\% de los votos, representando por lo tanto las opciones progresistas la mayoría absoluta de los votos emitidos por los expertos.

\section{Gráfico 5. Orientación ideológica del Plan Marco de Competitividad del Turismo Español - FUTURES I (PSOE)}

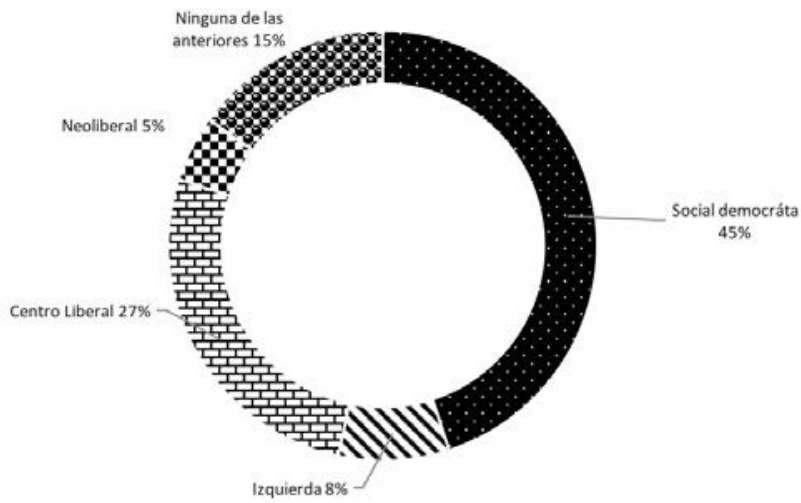

Fuente: elaboración propia

Siguiendo con el análisis, se presentan ahora los resultados del Plan Integral de Calidad del Turismo Español (PICTE) 2000-2006, elaborado por el gobierno del Partido Popular presidido por José María Aznar. Evaluando los resultados, la suma de las opciones ideológicas liberales (centro liberal + neoliberal) suponen un $46 \%$ de los votos, lo que representa un empate con la suma de las ideologías progresistas (social-demócrata + izquierda) que también suponen un $46 \%$ de las respuestas, por lo que puede afirmarse que se trataría del plan de turismo más moderado de todo el análisis (ver Gráfico 6).

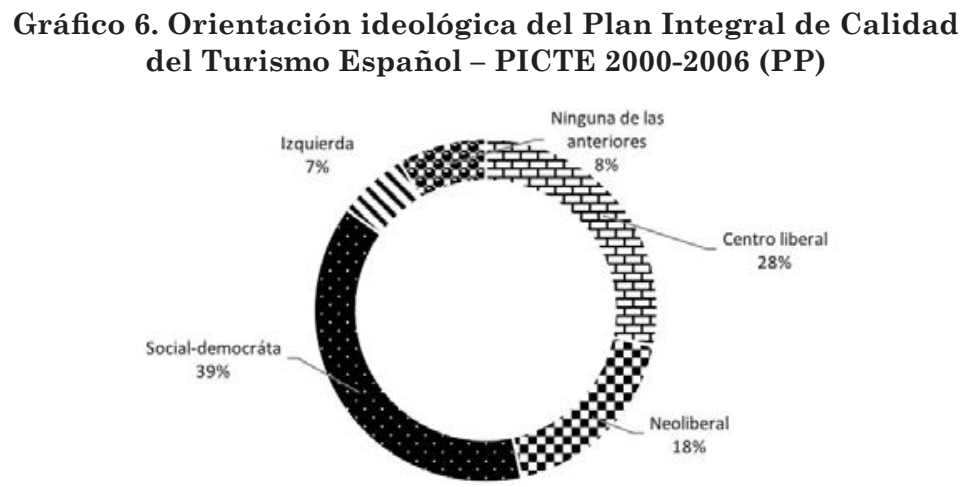

Fuente: elaboración propia 
A continuación, se presenta el análisis de los resultados obtenidos para el Plan del Turismo Español Horizonte 2020. De esta forma, la ideología social-demócrata representa por sí misma un 51\% de los votos totales emitidos, por lo que ya obtiene la mayoría absoluta de la orientación ideológica de este plan estratégico de turismo (ver Gráfico 7). Además, si sumamos los votos emitidos a la opción de izquierda, que representan el $10 \%$ del total, el resultado nos demuestra que el $61 \%$ del contenido del Plan Horizonte 2020 tiene una orientación ideológica progresista, lo que se encuentra en consonancia con la ideología del Partido Socialista Obrero Español (PSOE).

\section{Gráfico 7. Orientación ideológica del Plan del Turismo Español Horizonte 2020 (PSOE)}

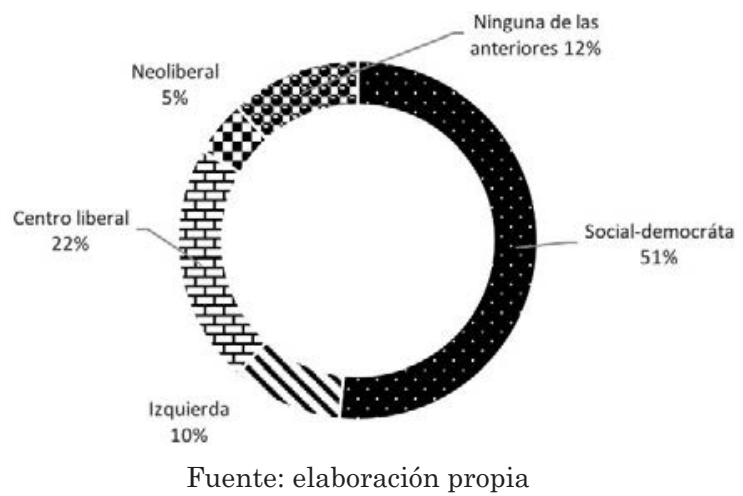

Finalmente, se analizan los resultados de los votos emitidos respecto al Plan Nacional e Integral de Turismo 2012-2015 (PNIT), aprobado bajo el mandato del Partido Popular en 2012. En este caso, la opción ideológica más votada ha sido la de centro liberal con un $36 \%$ de las respuestas totales. Adicionalmente, si añadimos a esta opción la neoliberal, que supone un 15\% de las respuestas, obtenemos que un 51\% de las respuestas (mayoría absoluta) efectuadas por los expertos en relación con este documento de política turística corresponden a las ideologías liberales, lo que se encuentra en consonancia con la orientación ideológica del Partido Popular (ver Gráfico 8).

\section{Gráfico 8. Orientación ideológica del Plan Nacional e Integral de Turismo 2012-2015 - PNIT (PP)}

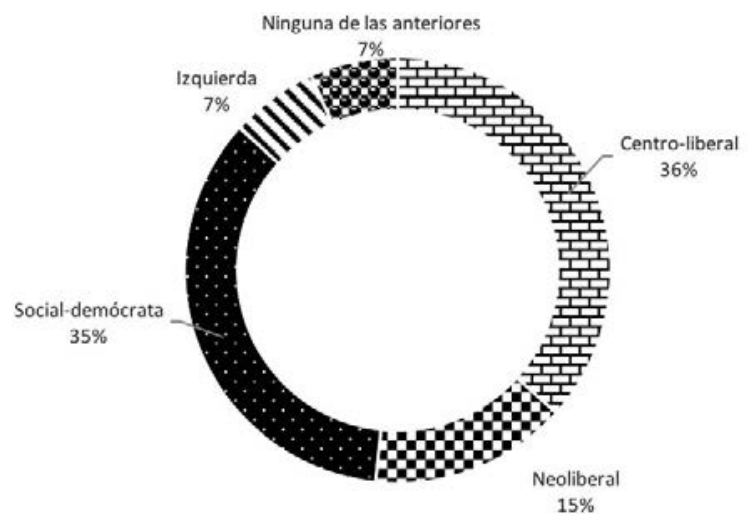

Fuente: elaboración propia

Una vez presentados los resultados de la primera fase de la Metodología Delphi, el estudio se centra ahora en los resultados cualitativos de la segunda fase. De esta forma, en cuanto al Plan Marco de Competitividad del Turismo Español - Plan FUTURES I, se presentan los comentarios del consenso 
alcanzado sobre la relación entre bienestar social e ideología social-demócrata. Así, el Experto en Turismo 1 señala que "se habla de bienestar social y aunque eso debería abarcar también a los empresarios y emprendedores parece que se interpreta más hacia los asalariados y personas en riesgo, por tanto, se interpreta como una frase de ideología socialista". Por su parte, el Experto en Turismo 4 dice que la relación se debe "seguramente porque en teoría la ideología social demócrata siempre fija como objetivo principal el bienestar social de la población".

En referencia al Plan Integral de Calidad del Turismo Español - PICTE 2000-2006, se presentan ahora las reflexiones de los expertos sobre la relación entre rentabilidad e ideología neoliberal. Así, el Experto en Turismo 4 señala que la relación se debe porque "seguramente en teoría la ideología neoliberal defiende la consecución de este objetivo, de primar los beneficios y la rentabilidad como forma de lograr el interés general". Por su parte, el Experto en Turismo 2 dice expresamente que "el objetivo de la actuación es marcadamente neoliberal, ya que fundamenta la proyección del modelo y los fines deterministas, en la obtención exclusiva de rentabilidad de las inversiones; ignorando o menospreciando otros resultados y conclusiones como el empleo y la RSC".

Analizando los resultados del Plan del Turismo Español Horizonte 2020, se muestran los comentarios expresados acerca de la relación entre sostenibilidad e ideología social-demócrata. De esta manera, el Experto en Turismo 4 dice que la relación se debe "seguramente porque en teoría la ideología social demócrata siempre fija como objetivo la sostenibilidad". Por su parte, el Experto en Turismo 2 explica detalladamente cuáles son los contenidos del enunciado que motivan la mencionada relación cuando dice "a causa de la mención expresa a conseguir una serie de objetivos. Todos ellos más sensibles a las ideologías social demócratas: el ordenamiento de la capacidad de carga, la garantía de la calidad del medio natural y cultural, la integración y bienestar social, y el reequilibrio territorial".

Finalmente, en cuanto a los resultados cualitativos del Plan Nacional e Integral de Turismo 20122015 (PNIT), se muestran los comentarios que explican la relación entre el centralismo en política turística y las ideologías liberales y conservadoras. Así, el Experto en Turismo 4 dice que "todas las ideologías plantean conseguir este objetivo, pero seguramente los encuestados piensan que la liberal vela por el liderazgo centralizado en el gobierno nacional, frente a las izquierdas que priman más la descentralización". Igualmente, resulta muy interesante la opinión del Experto en Turismo 2 que señala que "el pensamiento que formula el enunciado, es absolutamente conservador desde el estado, en cuanto se enfrenta a la posibilidad de una participación liberal del sector privado, aunque controlada, manteniéndose las estructuras y principios de la sostenibilidad. Considero que nunca, el liderazgo para alinear las voluntades de actores y los recursos en un proyecto turístico común, debe ser asumido por el gobierno de la nación..."

Asimismo, como parte final del cuestionario cualitativo correspondiente a la segunda fase se realizó una pregunta abierta a los expertos sobre su opinión acerca de la relación final entre ideología y política turística, obteniendo tres respuestas que niegan dicha relación y una respuesta que sí la identifica. De todas ellas, se ha considerado particularmente relevante destacar el comentario del Experto en Turismo 2 que dice que "Hemos de hablar de políticas comunes. Aunque nuevamente se ha de destacar, que en apariencia la socialdemocracia busca objetivos y fines más sociales, y las ideologías centro liberales, anteponen por encima de cualquier objetivo, el mantenimiento empresarial y la ocupación de los factores productivos (trabajo e inversión). Situando en escala inferiores, fines tan importantes como los sociales. Pero también en apariencia, la filosofía liberal ha de defender la sostenibilidad. Por lo que habremos de encontrar identidad en los objetivos y en las políticas implementadas por las ideologías. Pero aquellas matizadas, especialmente, por el manejo desigual de los instrumentos en el marco y desarrollo de las políticas".

\section{Conclusiones}

Como primera conclusión del enfoque y contenido de las políticas turísticas, se puede afirmar, que existen una serie de elementos comunes en las políticas turísticas del Gobierno de España con independencia del partido político, destacando elementos como son el bienestar social, la sostenibilidad, la calidad, la cooperación y los conceptos mercantiles (ver Cuadro 3). 


\section{Cuadro 3. Contenidos comunes de la política turística española}



Fuente: elaboración propia

Sin embargo, y atendiendo al contenido esencial de los documentos estratégicos de turismo, existen, como segunda conclusión, diferencias en el enfoque y contenido de las políticas turísticas desarrolladas por el Partido Socialista (PSOE) y las políticas turísticas aprobadas por el Partido Popular (PP), dado que, si realizamos un análisis cruzado de las coincidencias de enfoques por partido político, sí que existen elementos comunes en sus políticas turísticas, tras haber analizado todos los resultados. De esta forma, los contenidos comunes de las políticas turísticas del PSOE son competitividad, bienestar social, conservación medioambiental y sostenibilidad, calidad, participación de stakeholders, cooperación, consenso y conceptos mercantiles. Por su parte, los contenidos comunes de las políticas turísticas del PP son calidad, conceptos mercantiles, sostenibilidad, iniciativa y necesidades privadas, empleo, bienestar social y cooperación (ver Cuadro 4).

\section{Cuadro 4. Enfoques diferenciales de las políticas turísticas de PSOE y PP}



Fuente: elaboración propia

Asimismo, habiendo establecido los contenidos comunes de las políticas turísticas del PSOE y el PP, y los contenidos comunes de la política turística española, se pueden extraer ahora los elementos diferenciales de las políticas turísticas de cada partido político, es decir, aquellos elementos que son solamente propios de las políticas en turismo desarrolladas por un partido concreto y que diferencian su enfoque y visión del turismo de su oponente político. De esta manera, en el caso del PSOE los elementos serían la competitividad, la participación de stakeholders y el consenso, y en el caso del 
PP los elementos diferenciales serían la iniciativa privada, los intereses y necesidades privadas y el fomento del empleo (ver Cuadro 5).

Cuadro 5. Elementos diferenciales de las políticas turísticas de PSOE y PP

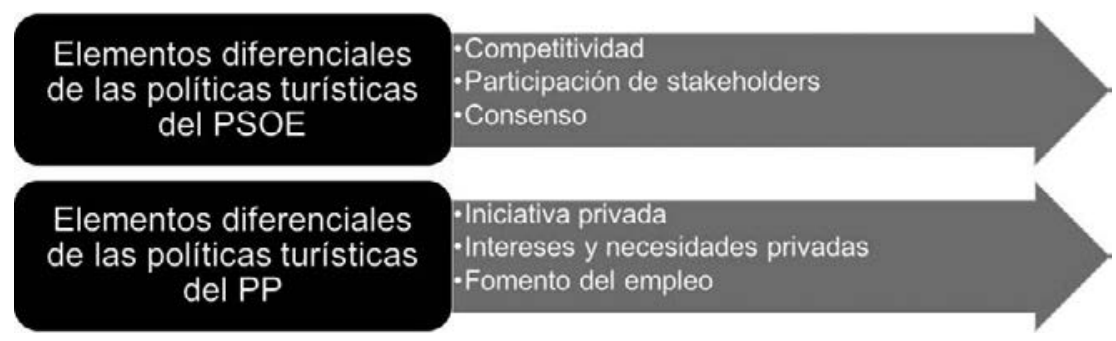

Fuente: elaboración propia

En referencia a las conclusiones de la orientación ideológica de las políticas turísticas, se puede extraer como primera conclusión, que todas las ideologías están presentes a lo largo del histórico de la política turística española y que las tendencias ideológicas de cada plan de turismo no parecen indicar la aplicación dogmática de una sola ideología al plan de turismo correspondiente. No obstante, si se analizan los resultados con detalle, si se puede extraer como segunda conclusión, que salvo en el Plan PICTE para el cual existe un empate, la orientación ideológica de los planes de turismo resultante del cuestionario sí indica una tendencia coincidente con la ideología del partido político que elaboró el documento.

El análisis de las respuestas cualitativas de los expertos consultados correspondientes a la segunda fase de la Metodología Delphi, muestra un cierto consenso de que el análisis de políticas turísticas y su ideología por medio de enunciados puede inducir a un sesgo y error, consistente en una tendencia a vincular todos los conceptos relacionados con la sostenibilidad y el bienestar social a una ideología social-demócrata; y por otro lado, vincular todos los conceptos relacionados con el mercado y la iniciativa privada con ideologías liberales. Sin embargo, sí que existe un experto que identifica una relación entre ideología y política turística que se refleja en el Cuadro 6.

\section{Cuadro 6. Ámbitos específicos de la relación entre ideología y política turística}

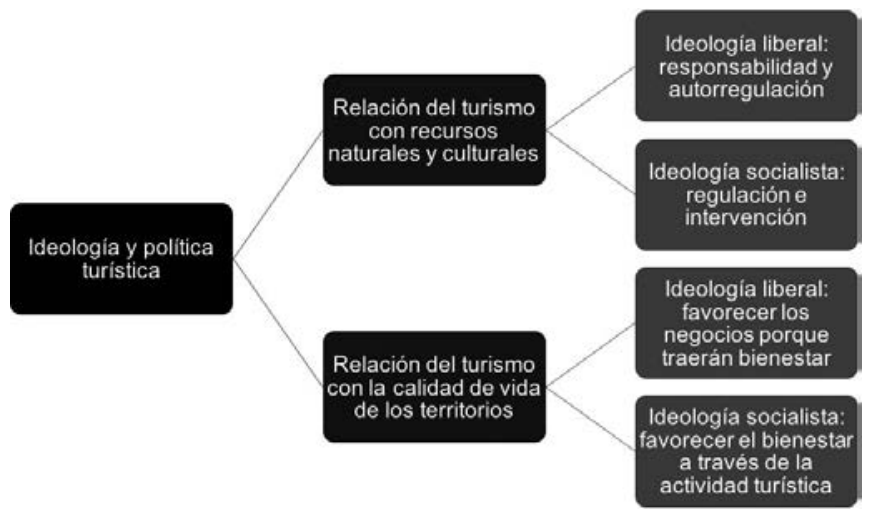

Fuente: elaboración propia, a partir de los resultados de la segunda fase de la Metodología Delphi correspondiente al Experto en Turismo 1

La conclusión final del proyecto de investigación es que existen diferencias importantes en los enfoques $y$ contenidos de las políticas turísticas de los diferentes partidos políticos. No obstante, igualmente existen una serie de contenidos comunes en el histórico de la política turística española con independencia del 
partido que ostenta el gobierno. Por su parte, en relación con las ideologías, no puede establecerse una relación generalizable entre ideología y política turística, pero sí que puede afirmarse que los partidos políticos aplican sus orientaciones y visiones sobre el turismo a las políticas turísticas que desarrollan. Por lo tanto, se podría hablar de una confirmación parcial de la hipótesis de la investigación.

\section{Agradecimientos}

Quiero agradecer especialmente la finalización de este estudio al Catedrático de Turismo de University of Surrey Prof. David Airey, quien me ayudó a contextualizar el proyecto de investigación y me sugirió mejoras y soluciones en todo momento, de lo que fue la primera versión de este proyecto. Asimismo, todo mi más sincero agradecimiento a la Profesora y Doctora Marta Plumed Lasarte, quien, con una dedicación incondicional, me ha ayudado hasta el final en el desarrollo y finalización de esta investigación, estando siempre atenta a mis dudas y preguntas y encontrándose siempre a disposición de mis solicitudes e inquietudes. Igualmente, quiero agradecer la participación en este estudio a los cinco expertos en turismo y en otras áreas de conocimiento que han formado parte del panel de expertos del Método Delphi. Ellos son David Mora Gómez, Andrés Fernández Alcantud, Enrique Martínez Cantero, Carlos Romero Dexeus y Manuel Figuerola Palomo, quienes han sido en su mayoría profesores míos durante el Máster Universitario en Turismo: Gestión de Empresas y Destinos Turísticos de la Universidad Antonio de Nebrija. Finalmente, también quiero agradecer la colaboración del Instituto de Turismo de España (Turespaña) por facilitar los documentos de política turística estatal que han sido necesarios para el desarrollo del estudio.

\section{Bibliografia}

Aguiló, E., Vich i Martorell, G.A.

1996. La investigación en el ámbito de la política turística. Revista de Estudios Turísticos, 129, pp. 23-35. Airey, D., y Chong, K.

2011. Tourism in China: policy and development since 1949. London: Routledge.

Almeida, F.

2012. Tourism Policy in Spain and Portugal. Cuadernos de Turismo, 30, pp. 287-289.

Correyero, B.

2004. La Administración turística española entre 1936 y 1951: el turismo al servicio de la propaganda política. Revista de Estudios Turísticos, 163-164, pp. 55-79.

Dredge, D. and Jenkins, J.

2007. Tourism planning and policy. Milton, QLD, Wiley.

Dye, R. T.

2008. Understanding Public Policy (12 ${ }^{\text {th }}$ edition). Englewood Cliffs, NJ: Pearson/Prentice Hall.

Elliot, J.

1997. Tourism: politics and public sector management. London: Routledge.

2016. Encuesta de Movimientos Turísticos en Fronteras (FRONTUR), Instituto Nacional de Estadística

Consultada el 02 de septiembre de 2016 en http://www.ine.es/jaxiT3/Tabla.htm?t=10835\&L=0

Fayos-Solá, E.

1996. Tourism policy: a midsummer night's dream? Tourism Management, 17 (6), pp. 405-412.

FEMP y SGT

2008. Modelos de gestión turística local. Principios y prácticas. Federación Española de Municipios y

Provincias y Secretaría General de Turismo.

Hall, C.M.

1998. Introduction to Tourism: development, dimensions and issues. South Melbourne: Addison, Welsley, Longman.

Hall, C.M. y Jenkins, J.

1995. Tourism and Public Policy. London: Routledge.

Harvey, D.

2007. Neoliberalism as creative destruction. Annals of the American Academy of Political and Social

Science, 610, pp. 22-44. 
Instituto Nacional de Estadística

2016. Notas de prensa, Disponible en http://www.ine.es/prensa/np975.pdf

Ivars, J.A.

2004. Tourism Planning in Spain: Evolution and perspectives. Annals of Tourism Research, 31 (2), pp. 313-333.

Ladd, E.C.

1989. The American Polity (3 ${ }^{\text {rd }}$ edition). New York: Norton.

Pearce, D.

1992. Tourist Organizations. Harlow: Longman.

Pearce, D.

1997. Tourism and the Autonomous Communities in Spain. Annals of Tourism Research, 24 (1), pp. 156-177.

Pellejero, C.

2004a. La política turística en España. Una perspectiva histórica. Las nuevas formas de turismo, Colección Mediterráneo Económico, 5, pp. 268-284.

Pellejero, C.

2004b. Organización administrativa e intervención del Estado en el sector turístico: 1951-1977. Revista de Estudios Turísticos, 163-164, pp. 81-97.

Secretaría de Estado de Turismo

2012. Plan Nacional e Integral de Turismo 2012-2015. Ministerio de Industria, Energía y Turismo.

Secretaria General de Turismo

1992. Plan Marco de Competitividad del Turismo Español (FUTURES I). Ministerio de Comercio y Turismo.

Secretaria General de Turismo

2000. Plan Integral de Calidad del Turismo Español. Ministerio de Economía.

Secretaría General de Turismo

2007. Plan del Turismo Español Horizonte 2020. Ministerio de Industria, Turismo y Comercio.

Velasco, M.

2004. Administración y política turística de 1978 a 2005. Revista de Estudios Turísticos, 163-164, pp. 99-122.

Velasco, M.

2005. ¿Existe la política turística? La acción pública en materia de turismo en España (1951-2004).

Política y Sociedad, 42 (1), pp. 169-195.

Wang, D. and Ap, J.

2013. Factors affecting tourism policy implementation: a conceptual framework and a case study in

China. Tourism Management, 36, pp. 221-233.

Webster, C. e Ivanov, S.

2016. Political ideologies as shapers of future tourism development, Journal of Tourism Futures, (Inédito). 